\title{
Surface Periodic Poling in Lithium Niobate and Lithium Tantalate
}

\author{
A. Busacca, M. Cherchi, S. Riva Sanseverino \\ Dipartimento Ingegneria Elettrica Elettronica e delle Telecomunicazioni, Università di Palermo \\ Viale delle Scienze - 90128 Palermo, ITALY \\ A. C. Cino, A. Parisi \\ CRES - Centro per la Ricerca Elettronica in Sicilia \\ Via Regione Siciliana 49 - 90046 Monreale (PA), ITALY \\ G. Assanto, M. Cichocki, \\ NooEL-Nonlinear Optics and OptoElectronics Lab. University Roma Tre \\ Via della Vasca Navale 84, 00146 Roma, ITALY \\ F. Caccavale, D. Calleyo, A. Morbiato \\ SAES Getters S.p.A., Viale Italia, 77, 20020 Lainate (Milano) ITALY
}

\begin{abstract}
Periodic Poling of Lithium Niobate crystals (PPLN) by means of electric field has revealed the best technique for finely tailoring PPLN structures and parameters, which play a central role in many current researches in the field of nonlinear integrated optics.

Besides the most studied technique of bulk poling, recently a novel technique where domain inversion occurs just in a surface layer using photoresist or silica masks has been devised and studied. This surface periodic poling (SPP) approach is best suited when light is confined in a thin surface guiding layer or stripe, as in the case of optical waveguide devices.

Also, we found that SPP respect to bulk poling offers two orders of magnitude reduction on the scale of periodicity, so that even nanostructures can be obtained provided an high resolution holographic mask writing technique is adopted. We were able to demonstrate $200 \mathrm{~nm}$ domain size, and also good compatibility with alpha-phase proton exchange channel waveguide fabrication.

Our first experiments on Lithium Tantalate have also shown that the SPP technology appears to be applicable to this crystal (SPPLT), whose properties can allow to overcome limitations such as optical damage or UV absorption still present in PPLN devices.
\end{abstract}

Finally, the issue of SPP compatibility with proton exchange waveguide fabrication will be addressed.

\section{INTRODUCTION}

The fast growing of optical communication systems requires all optical networks featuring devices like all optical switches and wavelength converters.

Optical parametric interactions in ferroelectric Lithium Niobate (LN) crystals have already been exploited for the realization of nonlinear devices suitable for all optical networks. In particular proton exchange (PE) waveguide technology together with periodic poling of ferroelectric domains of LN make available very efficient frequency doublers that rely on quasi phase matching (QPM) between the infrared (IR) pump and the generated second harmonic field [1].

Our attention has been focused on a new poling technique for LN crystals: the surface periodic poling of Lithium Niobate (SPPLN). With this technique only a tens of microns thick layer is poled and, on the other hand, it is possible to achieve very small periods with respect to standard bulk poling. In fact submicron poling has been demonstrated [2], [3], which is needed in some parametric interaction schemes, still unexplored due to technological limits.

In order to get good conversion efficiencies high optical power densities are required along the whole path of nonlinear interaction between the input pump radiation and the generated second harmonic radiation. This is why it is convenient to fabricate the SPPLN structures on channel waveguides that confine optical power on an area comparable with the square of the optical wavelength and are not affected by diffraction problems when propagating over centimeters interaction lengths.

The photolithographic mask for channel waveguide fabrication must withstand the proton exchange process, that requires dipping of the sample in an acid bath at high temperatures $\left(300^{\circ} \mathrm{C}\right)$ [4]. For this purpose we have patterned silica films that we have deposited using the Ion Plating Plasma Assisted (IPPA) technique on the $-\mathrm{Z}$ face of the LN samples. Patterning has been done with a standard photolithographic technique, followed by an anisotropic dry plasma etching [5].

On the other hand the fabrication of periodic gratings for SPPLN requires a photolithographic mask featuring a good uniformity and high dielectric constant, capable of withstanding the high electric field that is applied during the poling process. Using IPPA deposited silica we have been able 
to pattern micron period gratings [6], but standard lithographic techniques are not suitable for sub-micrometric patterning. This is why we have turned to patterning of photoresist masks with the Lloyd mirror, an holographic technique that can reach hundreds of nanometer period gratings [7].

In the following sections, we will show that in our experimentation we have developed a technologic process to ensure full compatibility between the $\mathrm{PE}$ waveguide fabrication technology on LN and the new promising SPPLN technique for the fabrication of nonlinear micron- and nanoscale period gratings.

\section{Masks ANd Optical Waveguides Fabrication}

Deposition of silica films has been performed using a thermal evaporator equipped with a power radiofrequency generator. This allow the deposition of dielectric thin film with the IPPA technique. During the whole evaporation process, the $\mathrm{LN}$ substrate to be covered is dipped in a reactive plasma, continuously bombarded by both inert and reactive particles. These particles transfer a high kinetic energy to the deposited film with the net effect of creating an oxidant and reactive environment. This allow to deposit non-stoichiometric silica layers with good substrate adhesion, high density and very good uniformity.

This system allows to deposit high quality silica layers with thickness ranging between $20 \mathrm{~nm}$ and $50 \mathrm{~nm}$ on $500 \mu \mathrm{m}$ thick LN substrates. The quality of the deposited films is proven by its capability of withstanding both the PE process and the poling process [5].

Since silica quality and compactness are very important parameters for both SPPLN and PE waveguide fabrication, first of all we have characterized density, optical properties and dielectric properties of deposited films.

Density has been evaluated with an accurate study of the etching rates in a hydrofluoric acid aqueous solution $\left(\mathrm{HF}: \mathrm{H}_{2} \mathrm{O}\right.$ $=1: 10$ ). In this way we have optimized the deposition process parameters and we have found a good and stable working region when the argon pressure is kept between $5^{*} 10^{-3}$ and $5^{*} 10^{-4}$ Torr and the radiofrequency power is chosen between 200 and $400 \mathrm{~W}$. In particular the best working point corresponds to $5^{*} 10^{-3}$ Torr argon pressure, $1.5^{*} 10^{-3}$ Torr oxygen pressure and $300 \mathrm{~W}$ radiofrequency power, leading to a $10 \mathrm{~nm} / \mathrm{min}$ film deposition velocity.

Optical characterization has been performed with the aid of a single prism coupling system, revealing the so called "darkmodes". Experimental data has been interpreted using the inverse WKB method that allows to estimate the thin film refractive index. It turns to be very close to 1.46 (that is the refractive index of bulk $\mathrm{SiO}_{2}$ ), and this confirms that this technique can produce high density films with good optical properties.

After film deposition on the $-\mathrm{Z}$ face of the LN substrate, a standard photolithographic approach together with an anisotropic plasma etching has been used to pattern both waveguides and periodic structures on the silica film. In particular the mask to realize the dielectric periodic pattern to be used in the SPPLN process, features a collection of $1 \mathrm{~cm}$ long and $1 \mathrm{~mm}$ wide gratings with periods ranging from 1.5 $\mu \mathrm{m}$ and $15 \mu \mathrm{m}$.

Pattern transfer from the photoresist to the silica layer has been achieved through an anisotropic etching process in which the sample lies on the negative electrode while a argon ion plasma is induced the vacuum chamber of the evaporation system at $5 * 10^{-4}$ Torr pressure. The argon ions, accelerated by a $400 \mathrm{~V}$ continuous voltage, bombard the silica layer passing through the windows previously open on the photoresist and physically selectively remove the underlying $\mathrm{SiO}_{2}$ film. In this way it is possible to etch $20 \mathrm{~nm}$ of silica in half an hour. Even though dry etching is a very slow process, it is preferable to the standard cheaper wet etching in hydrofluoric acid for two main reasons. First, because wet etching does not guarantee a good control of lateral etching, that is the well known problem of undercut. This prevent the realization of short (less than $2 \mu \mathrm{m}$ ) period gratings. Second, because hydrofluoric acid etching is not compatible with the poling process. In fact the etching rate depends on the domain polarization orientation (indeed this is the method that is commonly used to reveal the pattern of a poled sample).

This is why it is not possible to use hydrofluoric acid to remove the periodic mask after poling and a plasma dry etching must be chosen for the fabrication of the optical waveguides. The waveguide mask features a collection of 2-3 $\mathrm{cm}$ long waveguides with width ranging from $2 \mu \mathrm{m}$ to $18 \mu \mathrm{m}$. After mask patterning the waveguides are fabricated at $300{ }^{\circ} \mathrm{C}$ by dipping the sample in a sealed ampoule containing a benzoic acid with $3 \%$ lithium benzoate melt [4]. During the diffusion of $\mathrm{H}^{+}$ions within the crystalline matrix they substitute the $\mathrm{Li}^{+}$ions that are spread outside. This process increases the extraordinary refractive index and lowers the ordinary refractive index in the exposed superficial area. The resulting guiding layer features a graded index contrast profile with a maximum value about 0.015 at a wavelength of 632.8 $\mathrm{nm}$, and its depth is proportional to the square root of the proton exchange time. For our purposes we have fabricated 3 $\mu \mathrm{m}$ deep $\alpha$-phase PE waveguides to ensure monomodality at a wavelength of $1550 \mathrm{~nm}$ [8]. Using a BPM mode solver we have also simulated the modal shape of these modes and we have found a modal depth of the order of $10 \mu \mathrm{m}$ (Fig. 1), that is compatible with the domain depth we have obtained so far. This is an important point when considering the impact on the efficiency of a good overlap between the periodic grating and the propagating field. In principle this prove the compatibility between the SPPLN technique and the PE waveguides in LN.

Submicron grating have been written on a photoresist mask with an interferometric pattern obtained with the $441.6 \mathrm{~nm}$ light of a He-Cd laser. The samples have been covered by a 


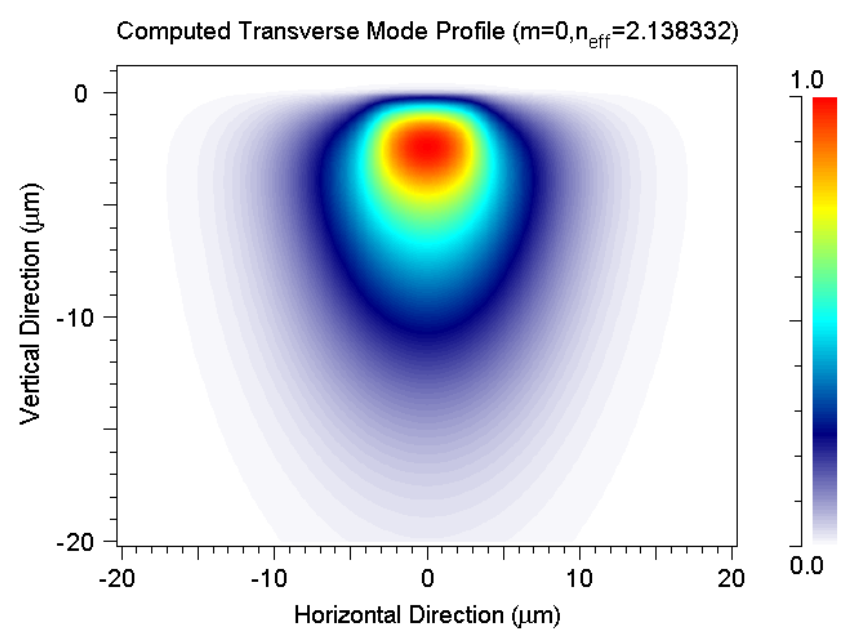

Fig. 1. Simulated TM mode profile (Electric field amplitude, arbitrary units) for a waveguide obtained with a $6 \mu \mathrm{m}$ wide mask.

400nm thick spin coated photoresist film (Shipley S1805) and have been set in the Lloyd configuration with a mirror orthogonal to them (Fig. 2). The He-Cd $6 \mathrm{~mW}$ beam it is expanded, collimated and then sent to the sample-mirror system, forming interference fringes with period given by $\lambda /[2 \sin (\theta)]$, being $\theta$ the rotation stage angle. The exposure time is about $15 \mathrm{~s}$. Since LN is transparent in the near UV we have also covered the back face of the samples with a matching index liquid in order to minimize the detrimental effects of multiple reflections in fringe visibility. After exposition, photoresist development has been checked through the grating diffraction efficiency under $\mathrm{He}-\mathrm{Ne}$ laser illumination. In this way we have been able to produce good quality $400 \mathrm{~nm}$ period gratings.

\section{PERIODIC DOMAIN INVERSION}

The technique for surface domain inversion is based on Electric field (E-field) poling at room temperature. The procedure is as follows: the $-\mathrm{Z}$ face of the crystal is covered with a periodic insulating layer photolithogra-phically patterned in order to achieve the appropriate E-field contrast

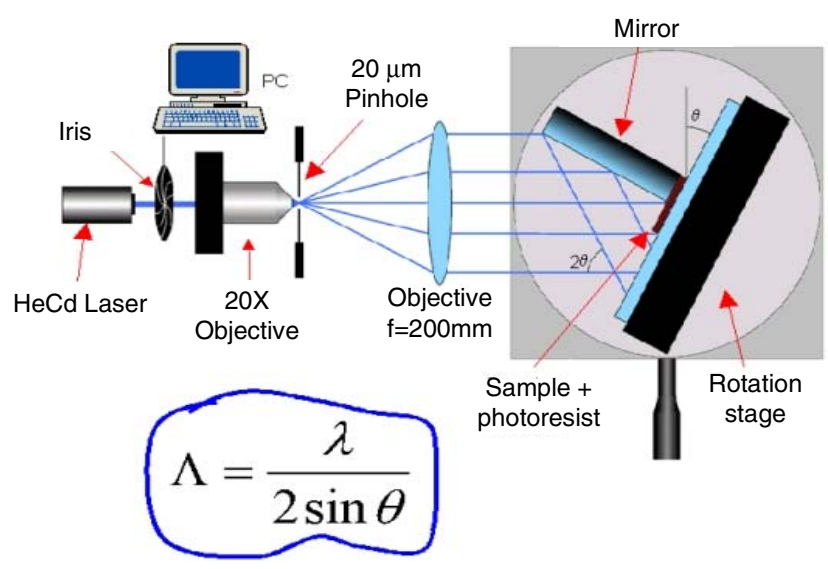

Fig. 2 Lloyd mirror configuration for interferometric writing of submicrometric gratings. which is necessary for spatially selective domain inversion. Both $\mathrm{Z}$ faces are then covered with conductive gel electrodes and a single high voltage (HV) pulse is applied across the 500 $\mu \mathrm{m}$ sample. The value of the HV varies with the thickness of the sample but the applied electric field must be of order 22 $\mathrm{kV} \mathrm{mm}^{-1}$ [9]. High-voltage was applied through conducting gel electrodes on both the uniform and the patterned "-Z" crystal faces, using $1.5 \mathrm{kV}$ square pulses of $500 \mathrm{~ms}$ on a $10 \mathrm{kV}$ offset to overcome the coercive field. Monitoring of both voltage and current was carried out during poling.

SPP relies on overpoling, i.e. the spreading of inverted ferroelectric domains and their merging beneath the lithographically-patterned mask. SPP can be described in terms of an electric field inversion when trapping of charges under the insulating layer are considered.

Domain depth and width are a function of the HV pulse length, of the poling period and of the mask duty cycle. It seems that HV pulse duration influences the domain width and in particular, when the pulse length increases, the mark-tospace ratio goes into the 50:50 direction.

To reveal the periodic ferroelectric pattern, after poling we etched the sample in an acid HF-bath. The different HF attach velocities in the $\mathrm{LN}-\mathrm{Z}$ and $+\mathrm{Z}$ face allow to reveal the crystal orientation. Fig. 3 is a scanning electron micrograph showing a mark-to-space ratio between poled and unpoled areas close to 50:50. The latter is ideal to exploit efficient nonlinear interactions in quasi-phase-matched LN.

\section{Results and Applications}

Standard bulk poling techniques work properly for grating periods on the order of ten microns. This is due to defects and unevenness in the whole volume of the samples. On the other hand, when working with PE optical waveguides light is confined in the surface of the samples and SPP must be preferred to bulk poling. After a systematic study of the PE waveguide fabrication with different tuning of the process parameters we have found the best working point to effectively superimpose the channel waveguides to the SPP grating. This can be seen in the SEM picture shown in Fig. 4 (where the domain pattern has been made visible by etching with hydrofluoric acid).

In our work we have also reached the world record of 400 $\mathrm{nm}$ poling period gratings as can be seen in the SEM photograph in Fig. 5.

These submicron periods are indispensable for the

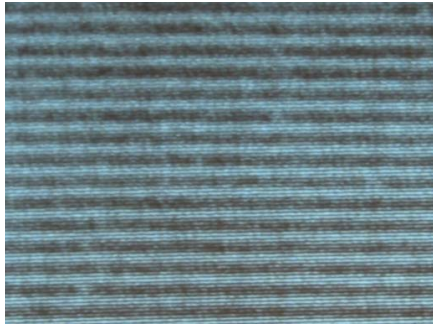

Fig. 3. SPPLN structure with $1.5 \mu \mathrm{m}$ period and 50:50 mark to space ratio. 


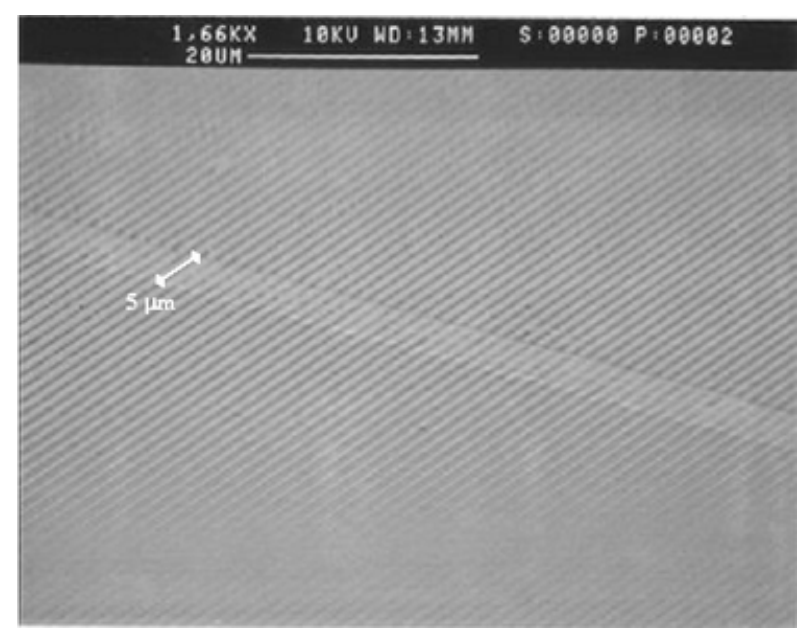

Fig. 4. PE channel waveguide on LN with SPP grating

realization of integrated optical devices based on collinear propagation of counter-propagating waves: this is the case of Backward Second Harmonic Generation (BSHG) or of the three wave mixing in a Counter-Propagating Parametric Amplifier (COPA) [10]. For example in Fig. 6 it is shown the COPA configuration proposed in Ref. [10], where the high power pump with wavelength $\lambda_{3}$ around $800 \mathrm{~nm}$ can be used to amplify the small signal with wavelength $\lambda_{1}$ around $1.55 \mu \mathrm{m}$, but with high efficiency only when the poling period $\Lambda$ is small enough to guarantee first order quasi phase matching. This implies SPP periods on the order of $200 \mathrm{~nm}$ when considering BSHG and on the order of $400 \mathrm{~nm}$ when dealing with COPAs. These values can be reasonably obtained with our technique.

\section{First EXPERIMENTS on Lithium Tantalate}

Another promising ferroelectric material for nonlinear applications is Lithium Tantalate (LT). Since in LT transparency goes down to $280 \mathrm{~nm}$ and photorefractive damage is lower than in $\mathrm{LN}$, it seems the natural candidate for UV SHG that requires grating periods on the order of $1.5 \mathrm{um}$. This is why we are now working on the extension of our SPP technique to this material (SPPLT).

Our first experiments of surface poling on $500 \mu \mathrm{m}$ thick

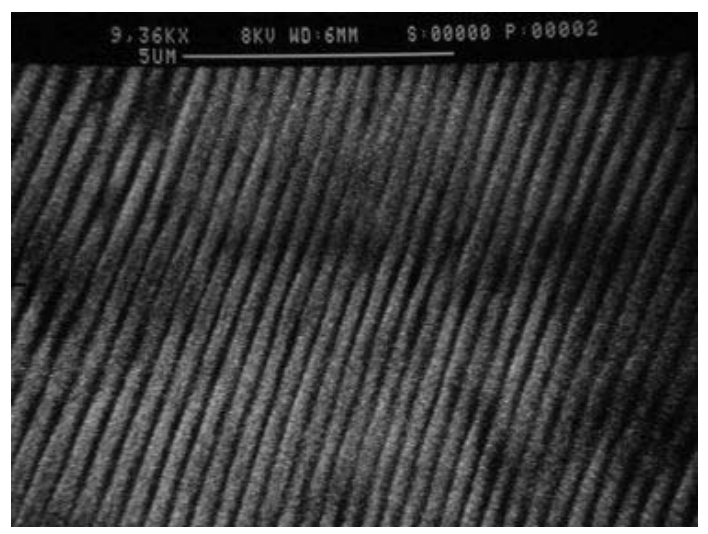

Fig. 5. SPP grating with $400 \mathrm{~nm}$ period.

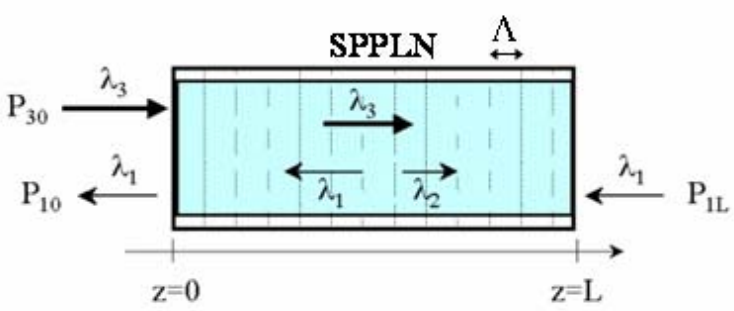

Fig. 6. Three-wave interaction in the COPA configuration

congruent LT samples from Saes Getters have been carried out by means of photoresist poling masks (Shipley S1813).

Masks were created on the $-\mathrm{Z}$ face of the crystal, following standard photolithographic technique, and they were composed of $4 \mu \mathrm{m}$ gratings with lengths up to $15 \mathrm{~mm}$. Gratings were oriented along the $\mathrm{X}$ crystal axis. Poling periods of such a dimension can be employed to obtain effective second harmonic conversion in the UV region by exploiting low order QPM interaction. To invert LT crystal domains we adopted the same setup used for LN, with the same conducting gel deposited on the surfaces of the sample to be poled so that it fills the features of the poling mask and acts as the electrodes where the high voltage poling pulse can be applied. Even though LN and LT have a different coercive field value, we observed the same poling pulse shapes for both materials, in particular we worked with $1.3 \mathrm{KV}$ pulse height and duration from 10 to $500 \mathrm{~ms}$ superimposed to a $10 \mathrm{KV}$ bias voltage. These waveforms applied across $500 \mu \mathrm{m}$ thick samples induce electric field values greater than the coercive fields of both materials. In Fig. 7 are plotted the voltage and current curves directly observed during the poling process. Integrating the current-time curve we estimated at about $3 \mu \mathrm{C} / \mathrm{mm}^{2}$ the electric charge flow within the crystal required to obtain a near complete inversion of ferroelectric domains, and thus surface periodic poling gratings of a good quality. Charge flow values only slightly reduced have been observed during the poling of LN crystal, what suggests that for this regard the two materials under study have a rather close behaviour. After poling, to put in evidence ferroelectric domains, we etched the samples in a $\mathrm{HF}$ acid bath for about $2 \mathrm{~h}$. Figure 8 is a scanning electron micrograph of a periodic poling grating on the $-\mathrm{Z}$ crystal surface.

It is worth noting that in contrast to what happens in bulk poling [11], where triangular shape domain walls inhibit the

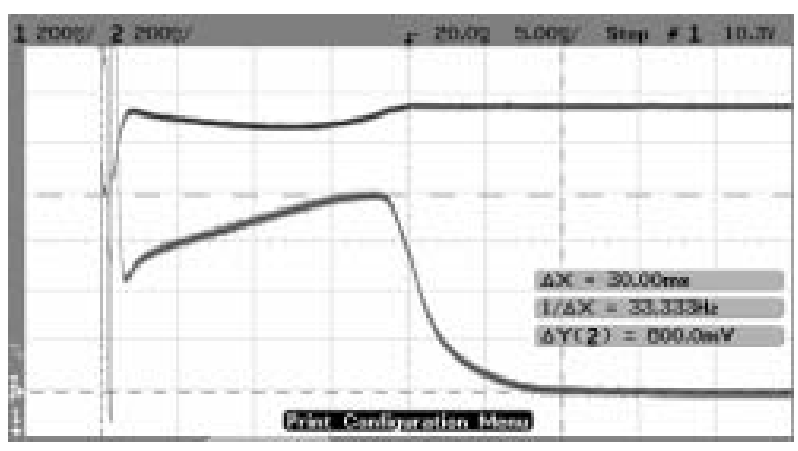

Fig. 7. Voltage (upper curve) and current measured during poling. 
formation of uniform grating lines, the SPPLT samples show a very uniform periodical structure along the all poled area. The triangular features can only be found at line edges.

For the SPPLT gratings it was found a typical 80:20 markto-space ratio between poled and unpoled regions. Considering that a good SHG conversion efficiency in the QPM configuration requires a 50:50 mark-to-space ratio, the following experiments are focused on finding the conditions to guarantee a better control of lateral domain size growth during poling.

\section{Conclusions}

In this work we found proper conditions to obtain SPPLN with grating periods from $15 \mu \mathrm{m}$ down to $400 \mathrm{~nm}$, and we proved its compatibility with $\alpha$-phase PE channel waveguides fabrication. We also started to experiment the extension of SPP technique to LT and reported our first results. The availability of micro- and nano-scale makes possible experimentations based on parametric interaction schemes which to date have not been explored due to technological limitations. Currently the produced devices are used for the experimental study of second harmonic generation in the near infrared with a counterpropagating configuration.

\section{AcKNOWLEDGMENT}

This research was supported by the Italian Ministero dell'Istruzione, dell'Università e della Ricerca, under a project funded within the Progetti di Ricerca di Interesse Nazionale 2003 (PRIN 2003) and coordinated by prof. Stefano RivaSanseverino.

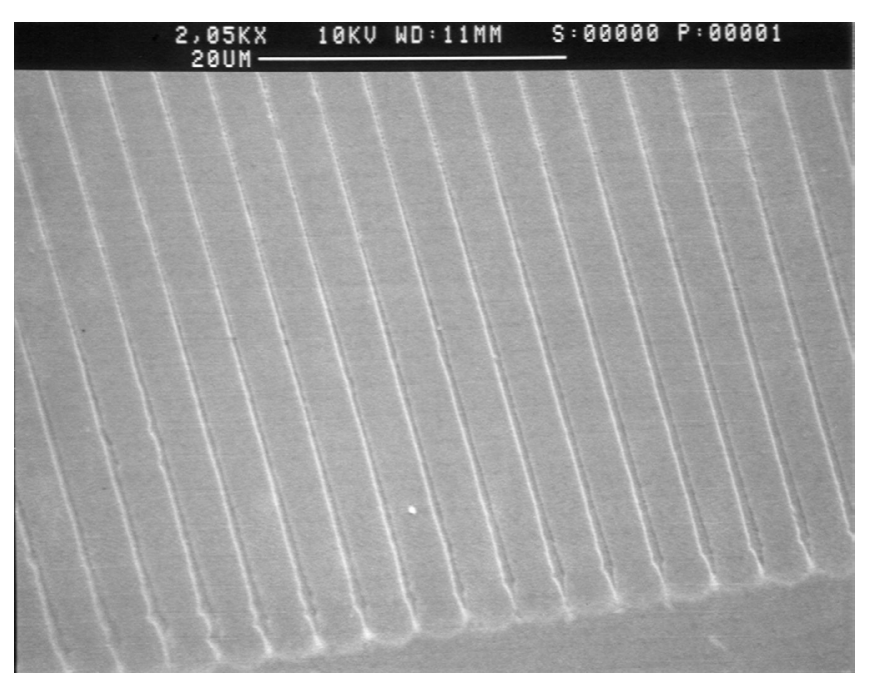

Fig.8. SEM image of a $4 \mu \mathrm{m}$ poling pattern on LT after HF etching.

\section{REFERENCES}

[1] M. M. Fejer, "Nonlinear Optical Frequency Conversion: Material Requirements, Engineered Materials, and Quasi-Phasematching", in Beam Shaping and Control with Nonlinear Optics, F. Kajzar and R. Reinisch, eds. New York: Plenum Press, 1998, pp 375-406.

[2] A. C. Busacca, C. L. Sones, V. Apostolopoulos, R. W. Eason, and S. Mailis, "Surface domain engineering in congruent lithium niobate single crystals: a route to sub-micron periodic poling", Appl. Phys. Lett., Vol. 81, pp. 4946-4948, Dec. 2002.

[3] A. C. Busacca, C. L Sones, R. W. Eason, and S. Mailis, "First-order quasi-phase-matched blue light generation in surface-poled Tiindiffused lithium niobate waveguides", Appl. Phys. Lett., Vol. 84, pp. 4430-4432, N. 22, May 2004

[4] P. Baldi, M. De Micheli, K. El Hadi, A. C. Cino, P. Aschieri, D.B. Ostrowsky, "Proton exchanged waveguides in $\mathrm{LiNbO}_{3}$ and $\mathrm{LiTaO}_{3}$ for integrated lasers and nonlinear optics", Opt. Eng., Vol. 37, No 4, pp. 1193-1202, Apr. 1998

[5] A. Parisi, A. C. Cino, A. C. Busacca, and S. Riva-Sanseverino, "Nonstoichiometric silica mask to fabricate reverse proton-exchange waveguides in lithium niobate crystals", Appl. Opt., Vol. 43, No 4, pp. 940-943, Feb. 2004

[6] A. C. Busacca, A. C. Cino, S. Riva-Sanseverino, M. Ravaro, and G. Assanto, "Silica masks for improved surface poling of lithium niobate", Electron. Lett., Vol. 41, N. 2, pp. 92-93, Jan. 2005

[7] A. C. Busacca, A. C. Cino, M. Ravaro, G. Assanto, F. Caccavale, A. Morbiato, and S. Riva Sanseverino, "Nano-domains definition in congruent lithium niobate by surface periodic electric-field poling", Proc. 12th European Conference on Integrated Optics ECIO'05, Grenoble (France), 6-8 Apr. 2005

[8] L. Chanvillard, P. Aschieri, P. Baldi, D. B. Ostrowsky, and M. de Micheli; L. Huang and D. J. Bamford "Soft proton exchange on periodically poled $\mathrm{LiNbO}_{3}$ : A simple waveguide fabrication process for highly efficient nonlinear interactions", Appl. Phys. Lett., Vol. 76, N. 9, pp. 1089-1091, Feb. 2000.

[9] V. Shur, E. Rumyantsev, R. Batchko, G. Miller, M. M. Fejer, and R. Byer, "Physical Basis of the Domain Engineering in the Bulk Ferroelectrics", Ferroelectrics, Vol. 221, pp. 157-159, 1999

[10] K. Gallo, P. Baldi, M. De Micheli, D. B. Ostrowsky, and G. Assanto, "Cascading phase-shift and multivalued response in counterpropagating frequency nondegenerate parametric amplifiers", Opt. Lett., Vol. 25, No 13, p. 966-968, Jul. 2000

[11] J.-P. Meyn, C. Laue, R. Knappe, R.Wallenstein, M.M. Fejer, "Fabrication of periodically poled lithium tantalate for UV generation with diode lasers", Appl. Phys. B 73, pp. 111-114, 2001 